\title{
An Energy-Efficient Clustering Algorithm for Wireless Sensor Networks
}

\author{
FENG Cheng-Xu, LIU Zhong, LIU Zhi-kun and Fang Biao \\ Electronics Eng. College, \\ Naval Univ. of Engineering \\ Wuhan, Hubei, China \\ kerryfengcx@126.com
}

\begin{abstract}
In Wireless Sensor Networks (WSN), cluster networks structures have the advantage of providing scalable and energy-efficient solutions as the sensor nodes are highly energy-constrained. In this paper, we propose a dynamic clustering algorithm for WSN which is in application to event monitoring. In this clustering algorithm, the cluster head selection approach takes the received signal strength residual energy of node and distance between the cluster head and the sink node into consideration. Through this method, the cluster size is constrained and the energy consumption of intra-cluster communication is reduced. The simulation results show that the proposed algorithm can efficiently conserve energy and prolong the lifetime of WSN, especially when the sink node is far from the network.
\end{abstract}

Keywords- Wireless sensor networks, Cluster head, Energy efficient clustering, Network lifetime

\section{INTRODUCTION}

With the advances in technologies of micro-electro mechanical systems, embedding system technology and wireless communication with low power consumption, it is now possible to produce micro wireless sensors for sensing, wireless communication and information processing. These inexpensive and tiny sensor nodes work together to form a wireless sensor networks (WSNs).

It is well-known that a clustering architecture enables better resource allocation and helps to improve power control [1]. Dynamic cluster architecture offers desirable features such as survivability, scalability and formation of a cluster which is triggered by certain events of interest. Because of these features, dynamic cluster is suitable for the application of target tracking [2]. Although there has been a good deal of work on the development of static clustering algorithm [3], the dynamic clustering protocols has not been fully studied yet. A hierarchical dynamic clustering method is introduced in [4], but it causes high maintenance costs. In [5] a dynamic clustering method based on Voronoi diagram is studied, however it suffers from high computation degree and the cluster head $(\mathrm{CH})$ selection mechanism is not rationality. [6] takes both the node sensing information and the node energy for the $\mathrm{CH}$ selection in consideration, whereas it ignores the distance of the $\mathrm{CH}$ to the sink node and the cluster size is not restricted.

In this paper, an improved energy efficient dynamic clustering protocol based on [6] is proposed. It takes the distance of the $\mathrm{CH}$ to the sink node in consideration and restricts the cluster size by a new method. The rest of the paper is organized as follows. We introduce the network model, energy model and the signal decay model in section 2 . The issue description is also contained in this section. We delve into the protocol details in section 3 . Then the simulation results and evaluation of the performance is analyzed in section 4. Finally, we offer some conclusions in section 5 .

\section{MODELS AND ISSUE DESCRIPTION}

\section{A. Network Model}

The network model of the dynamic clustering algorithm we proposed is composed as follow:

1) All nodes in the network are homogenous except the sink node. These nodes have the same initial energy, ability of data aggregation, detection range, transmission range and the transmission range is much bigger than the detection range.

2) The transmit power can be adjusted in an available range.

3) The position of each node is fixed and is known .

4) There is only one sink node and it's immobile.

\section{B. Energy Model}

The energy consumption of the cluster member includes three phases. In cluster formation phase, the cluster member receives $\mathrm{CH}$ election information; When the cluster has been constructed, the cluster member sends event information to the $\mathrm{CH}$; When the lifetime of the cluster has ended, the cluster member receives reorganization message. The energy consumption of the $\mathrm{CH}$ is as follow: in cluster formation phase, the $\mathrm{CH}$ receives reorganization information from former $\mathrm{CH}$ and broadcasts an advertisement message (ADV) to the cluster member. When the cluster has been constructed, the $\mathrm{CH}$ receives event information from the cluster members and performs data aggregation. Then the $\mathrm{CH}$ transmits data to the sink node. When the lifetime of the cluster has ended, the $\mathrm{CH}$ broadcasts reorganization message.

Different assumptions have been made by researchers about the radio characteristics, including energy dissipation in transmit and receive modes. We assume that the energy consumption of the sensor node is mainly due to data transmission and reception. We use the same radio model as stated in [8]. Both the free space ( $d^{2}$ power loss) and the multi-path fading ( $d^{4}$ power loss) channel models are used in this paper, depending on the distance between the transmitter and receiver. Power control can be used to invert 
this loss by appropriately setting the power amplifier-if the distance is less than a threshold equation reference goes here $d_{0}$, the free space model is used; otherwise the multipath model is used. Thus, to transmit a $k$ bit message a distance $d$, the radio expends [8]:

$$
E_{T x}(k, d)= \begin{cases}k E_{\text {elec }}+k \varepsilon_{\text {fs }} d^{2} & d<d_{0} \\ k E_{\text {elec }}+k \varepsilon_{m p} d^{4} & d \geq d_{0}\end{cases}
$$

and to receive this message, the radio expends:

$$
E_{R x}(k)=k E_{\text {elec }}
$$

Where, $E_{\text {elec }}$ is the electronics energy; $\varepsilon_{f s}$ and $\varepsilon_{m p}$ are the amplifier energy of the free space model and the multipath model.

\section{Signal Decay Model}

This paper adopts the signal decay model of [9].The signal energy measure on the $i^{\text {th }}$ sensor node over a time interval $t$, denoted by $S_{i}(t)$, can be expressed as follows:

$$
S_{i}(t)=\frac{S}{r_{i}^{\alpha}(t)}+n_{i}
$$

In equation (3), $S$ is a scalar that denoting the signal strength emitted by the target; $r_{i}(t)$ is the distance between the $i^{\text {th }}$ sensor node and the target; $\alpha(=2)$ is an energy decay factor. $n_{i}$ is the measure noise.

\section{Issue Description}

An energy effective dynamic clustering algorithm should follow some principles as follows:

1) The signal energy measured on the sensor should be strong. A strong signal means the sensor is near to the target. It can get more credible information and the data aggregation can be more accuracy;

2) The $\mathrm{CH}$ should have more residual energy. The $\mathrm{CH}$ performs the task of maintain the cluster and inter-cluster communication. Thus, it will consume more energy.

3) The cluster size should be reasonable. It means that the cluster size should be neither too large nor too small. In a big size cluster, the communication between boundary cluster member and the $\mathrm{CH}$ will consume too much energy. Otherwise, too much cluster member may cause more collision. On the other hand, a small size cluster has a small detection range and the $\mathrm{CH}$ changes too frequently which also would cause additional energy consumption. Thus, it requires confining the cluster size in order to insure the locality of the cluster.

\section{CLUSTERING ALGORITHM}

The energy efficient dynamic clustering algorithm this paper proposed can be described as follow:

1) When the event of interest occurs in the detection area, the nodes which detected the target signal start to cluster, the clustering process includes two phases: $\mathrm{CH}$ selection and cluster member recruitment.
2) The cluster members transmit the information they have collected to the $\mathrm{CH}$.

3) The $\mathrm{CH}$ receives the information from its members and transmits the output of data aggregation to the sink node.

4) When the lifetime of the cluster ends, the $\mathrm{CH}$ broadcasts reorganization message and the nodes start to construct new cluster.

In what follows, we focus on describing cluster head selection and cluster member recruitment in detail.

\section{A. Cluster Head Selection}

When the event of interest is detected by some nodes in the network, the nodes whose detected signal strength exceeds a predefined threshold $S_{0}$ are selected to be the $\mathrm{CH}$ volunteers. Then they broadcasts their locations and signal strength values. Each volunteer sets a back-off timer. If volunteer $i$ does not receive a solicitation packet until the back-off timer expires, it becomes $\mathrm{CH}$ and broadcasts an information solicitation packet. Whether a volunteer can become a $\mathrm{CH}$ or not that is determined by the back-off timer. To design the back-off timer, we consider three factors: the detected signal strength, residual energy of node and the distance between $\mathrm{CH}$ to the sink node.

The back-off timer value determined by:

$$
\tau_{i}=\left(\alpha_{1}\left(\frac{s_{0}}{s_{i}}\right)+\alpha_{2}\left(1-\frac{q_{i}}{q_{0}}\right)+\alpha_{3}\left(\frac{d_{i}}{d_{\text {max }}}\right)\right) \tau_{\text {max }}
$$

$\alpha_{1}, \alpha_{2}, \alpha_{3}$ are the weighted coefficient. $\alpha_{1}+\alpha_{2}+\alpha_{3}=1$. $S_{i}$ is the detected signal strength of node $i . S_{0}$ is the predefined threshold of the detected signal strength. $q_{i}$ is the residual energy of node $i . q_{0}$ is the initial energy of node $i$. $d_{i}$ is the distance from node $i$ to the sink node. $d_{\max }$ is the maximum distance value from nodes in network to the sink node. The distances can be calculated through the location information of nodes. $\tau_{\max }$ is the maximum available clustering delay value. $\tau_{\max }$ denotes the maximum back-off time value because $s_{0} / s_{i} 、 q_{i} / q_{0} 、 d_{i} / d_{\max }$ are values in range $(0,1)$. The equation (4) shows that if node $i$ detected a stronger signal strength, has more residual energy and nearer to the sink node, its timer value will be smaller and it has a higher probability to become a $\mathrm{CH}$.

If $i$ does not receive a solicitation packet until the backoff timer expires, it becomes $\mathrm{CH}$ and broadcasts an information solicitation packet. The packet contains its ID, location and time information. If by the time the back-off timer expires, $i$ receives a solicitation packet from other volunteer, it cancels the timer and become a cluster member. It will store the relative information of the $\mathrm{CH}$ and synchronize its time to the $\mathrm{CH}$.

\section{B. Cluster Member Solicitation}

Many studies select the transmission range to be the cluster range [10][11]. The disadvantage is the locality of the cluster can not be achieved which may cause energy waste. Otherwise, if we set a specialized range for the cluster, the node number of the cluster may be not enough to carry out 
the task because of the random deployment of the nodes and the indeterminacy of the target location. This paper proposed an adaptive method to solve this problem. We firstly set a threshold range $\left[N_{\min } N_{\max }\right]$ for the nodes which detected the target. If the active nodes number in the cluster range satisfies $N_{a}<N_{\min }$, the active radius $R_{a}$ should be extended. The adjust radius is $R_{\text {adjust }}$. If $N_{a}>N_{\max }$, the active radius $R_{a}$ should be reduced. This adjusted process will be continued until $N_{a} \in\left[N_{\min } N_{\max }\right]$ and then we can determine the cluster radius $R_{a} . N_{\min }$ and $N_{\text {max }}$ can be determined by the task requirement in applications. The basic principle is ensuring the $\mathrm{CH}$ can collect enough replies from the cluster members in order to estimate the target state information.

Based on this method, the cluster range will be small when there are a lot of active nodes around the $\mathrm{CH}$ and the $\mathrm{CH}$ will select a big cluster range when the number of the active node is small. In a word, the $\mathrm{CH}$ can determine the cluster range adaptively through the method.

\section{SimUlation AND PERFORMANCE EVAluAtion}

In order to evaluate the new algorithm this paper proposed, we compare our algorithm with the dynamic clustering protocol in [6] in the same condition. The simulation is configured as network size of $200 \mathrm{~m} * 200 \mathrm{~m}$ with 200 nodes randomly distributed. Each node is equipped with a battery having $2 \mathrm{~J}$ initially. $\varepsilon_{\text {elec }}$ is $50 \mathrm{~nJ} / \mathrm{bit}, \mathcal{E}_{f \mathrm{~s}}$ is $10 \mathrm{pJ} / \mathrm{bit} / \mathrm{m} 2, \quad \varepsilon_{m p}$ is $0.0013 \mathrm{pJ} / \mathrm{bit} / \mathrm{m} 4, \quad d_{0}$ is $75 \mathrm{~m}$, the transmission data is $4000 \mathrm{bit}, N_{\min }$ is $10, N_{\max }$ is $15, \alpha_{1}$ is $0.3, \alpha_{2}$ is $0.5, \alpha_{3}$ is 0.2 , the signal source strength is 100 , the threshold $S_{0}$ is 0.5 .

In the $\mathrm{CH}$ selection phase, the algorithm we proposed takes the distance between the volunteer and the sink node into consideration in order to reduce the energy consumption when the $\mathrm{CH}$ transmit data to the sink node. In the experiment, we set the abscissa of the sink node to be $100 \mathrm{~m}$, and the ordinate of the sink node is selected from $300 \mathrm{~m}$ to $900 \mathrm{~m}$. It means the distance from the network to the sink node is increasing. The residual energy of the nodes is random values in range $(0,2)$. We compare the energy consumption when the $\mathrm{CH}$ transmits data to the sink node between the new algorithm and the algorithm in [6].

Figure1 shows that the energy consumption of the two protocols both increase when the distance from the network to the sink node increase. In each sink node position, the energy consumption generated by the dynamic clustering algorithm this paper proposed (protocol 1) is less than the algorithm of [6] (protocol 2). With the increase of the distance between the network and the sink node, algorithm 1 can saved more energy. The simulation result shows that the distance between the network and the sink node plays an important role in energy consumption. Then we compare the dead rate of nodes and the lifetime of the network of the two protocols, as figure2 shows. We set the sink node position to be $(100,350)$.

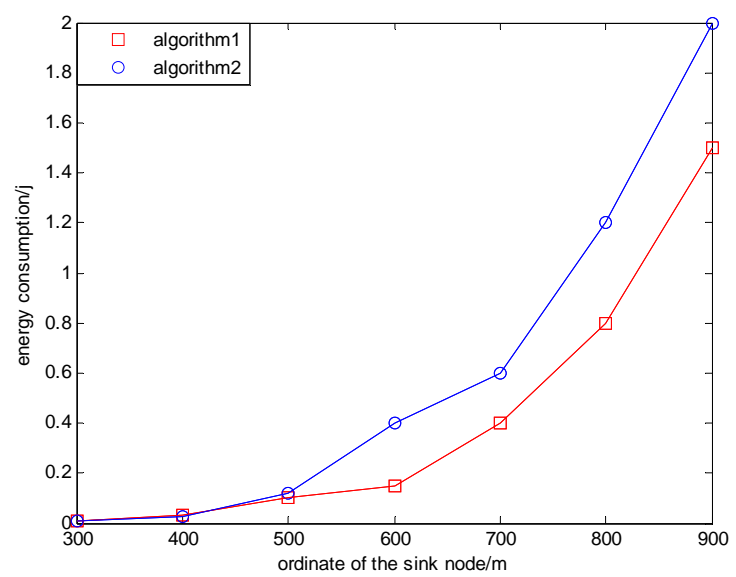

Fig. 1. Energy consumption of transition between $\mathrm{CH}$ and sink node

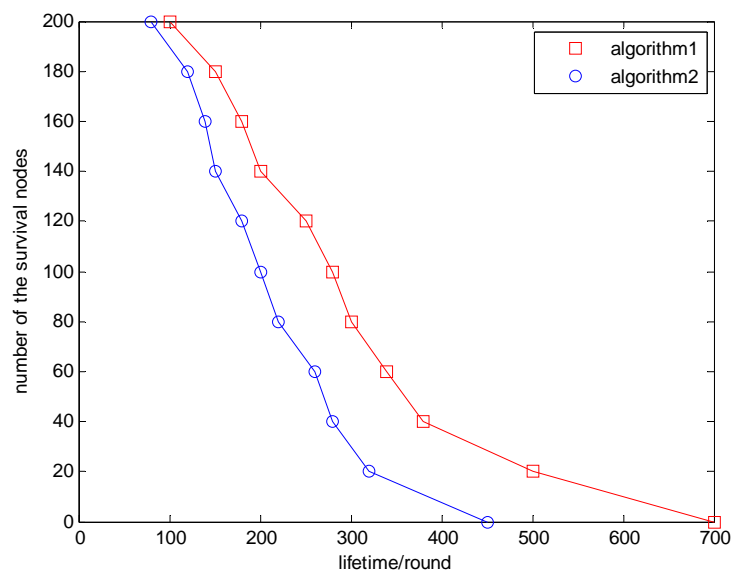

Fig. 2. Number of the survival nodes and lifetime of the network

Figure2 shows that the first dead node (FDN) occurs in the 80th round when protocol 1 is performed, and it occurs in the 100 nd round when protocol 2 is performed. Comparing with protocol 1, the FND is prolonged 36\% through protocol 2. The lifetime of the network is prolonged from 450 rounds to 700 rounds. The new protocol can effectively prolong the lifetime of the network. The performance of protocol 2 is better than protocol 1 because of two reasons: 1) protocol 2 takes the distance between the sink node and the $\mathrm{CH}$ into consideration which saves the energy of data transmission from the $\mathrm{CH}$ to the sink node. 2) as the cluster range is restricted, the data transmission between the cluster members and the $\mathrm{CH}$ causes less energy consumption.

\section{CONCLUSIONS}

An energy efficient dynamic clustering algorithm for wireless sensor networks is proposed in this paper. In the $\mathrm{CH}$ selection phase, the protocol takes the detected signal strength, the residual energy of node and the distance 
between the $\mathrm{CH}$ and the sink node into consideration. In the cluster member solicitation phase, the protocol restricts the size of the cluster based on the task and the cluster range could be adjusted adaptively. The protocol can efficiently conserve energy and prolong the lifetime of the network.

In this paper, we propose a dynamic clustering algorithm for WSN which is in application to event monitoring. In this clustering algorithm, the cluster head selection approach takes the received signal strength residual energy of node and distance between the cluster head and the sink node into consideration. Through this method, the cluster size is constrained and the energy consumption of intra-cluster communication is reduced. The simulation results show that the proposed algorithm can efficiently conserve energy and prolong the lifetime of WSN, especially when the sink node is far from the network. As future work, we intend to study the influence of the $\mathrm{CH}$ selection caused by the weighted coefficient.

\section{REFERENCES}

[1] Ming Yu, Kin K.Leung, Aniket Malvankar, “A Dynamic Clustering and Energy Efficient Routing Technique for Sensor Networks,” IEEE Transaction on Sensor Networks, vol. 6, no. 8, pp. 3069-3078, August 2007.

[2] J. C. Lin, G. J. Li, X. N. Zhou, et al, "DPM-based method for tracking maneuvering targets in wireless sensor networks," Journal on Communications, vol. 31, no. 12, pp. 90-96, Dec. 2010.
[3] H. Yang, B. Sikdar, "A protocol for tracking mobile targets using sensor networks,” Proc. IEEE Workshop Sensor Network Protocols and Applications, Piscataway, USA: IEEE Press 2003, pp. 71-81.

[4] W. Zhang, G. Cao, "DCTC: dynamic convoy tree-based collaboration for target tracking in sensor networks," . IEEE Transactions on Wireless Communications, vol. 3, no. 5, pp. 16891701, May 2004

[5] W. Chen, J. C. Hou, L. Sha, "Dynamic clustering for acoustic target tracking in wireless networks," IEEE Transactions on Mobile Computing, vol. 3, no. 3, pp. 258-271, Apr. 2004.

[6] K. B. Deng, Z. Liu, "Target tracking with dynamic clusters in wireless sensor networks,” ACTA ARMAMENTARII, vol. 29, no. 10, pp. 1197-1202, Dec. 2008.

[7] L. Chen, B. H. Zhao, "Data fusion oriented routing protocol based on LEACH," Journal of Beijing university of posts telecommunications, vol. 32, no. 5, pp. 71-74, May 2009.

[8] W. B. Heinzelman, A. P. Chandrakasan, H. Balakrishnan, "An application-specific protocol architecture for wireless microsensor networks," IEEE Transactions on Wireless Communications, nol..1, vol. 4, pp. 660-670, Apr. 2002.

[9] D. Li, Y. H. Hu, "Energy based on collaborative source localization using acoustic micro-sensor array,” Journal EUROSIP Applied Signal Processing, vol. 4, pp. 321-337, July. 2003.

[10] S. Selvakennedy, S. Sinnappan, S. Yi, “A biologically-inspired clustering protocol for wireless sensor networks,” Elsevier Computer Communications, vol. 30, no.10, pp. 2786-2801, Dec. 2007.

[11] X. N. Fan, Y. L. Song, "Improvement on LEACH protocol of wireless senor network," International Conference on Sensor Technologies and Application, vol.5, no. 30, pp. 260-264, Dec. 2007. 